\title{
ENSINO DE BIM: EXPERIÊNCIA EM PROJETO DE INSTALAÇÕES ELÉTRICAS NO IFRN
}

\author{
Lucas Gomes - lucasemanoel.souza@gmail.com \\ Universidade Federal do Rio Grande do Norte \\ Natal - RN
}

Thalita Costa - thalitagiesta@yahoo.com.br

Universidade Federal do Rio Grande do Norte

Natal $-R N$

Josyanne Giesta - josyanne.giesta@ifrn.edu.br

Instituto Federal de Educação, Ciência e Tecnologia do Rio Grande do Norte

Natal - RN

Alfredo Costa Neto-alfredo.neto@ifrn.edu.br

Instituto Federal de Educação, Ciência e Tecnologia do Rio Grande do Norte

Natal - RN

\section{Resumo}

O decreto federal $\mathrm{n}^{\circ} 10.306$ de 02 de abril de 2020 aumentou a urgência de implementação do Building Information Modeling (BIM) no mercado da área de Arquitetura, Engenharia, Construção e Operação (AECO) no país. Tal realidade implica diretamente na academia, que precisa atender na formação de profissionais em BIM. No entanto, os cursos da área já possuem matriz curricular com carga horária definida. Nesse contexto, a academia enfrenta a dificuldade de adequar a necessidade de inserção de conteúdos BIM, sem necessariamente comprometer os demais conteúdos. Diante desse quadro, as plataformas de aprendizagem a distância podem se apresentar como um aliado. $\mathrm{O}$ artigo tem como objetivo verificar a percepção de alunos do curso de Edificações do Instituto Federal de Educação, Ciência e Tecnologia do Rio Grande do Norte (IFRN) em experimentação de cenário educacional para inserção do BIM. O método de pesquisa adotado foi o estudo de caso, com recorte temporal no ano de 2019 e a unidade de análise foi o evento Clube do Instalador, do IFRN, que conta com a participação dos alunos do curso de Edificações. Os resultados indicam que o uso de ferramentas tecnológicas de ensino permite que jovens alunos inseridos neste cenário apresentem melhor desempenho na aprendizagem de atividades acadêmicas. Nesse contexto, pode-se concluir que a aplicação do cenário educacional proposto, utilizando Ambiente Virtual de Aprendizagem (AVA), tem potencial para facilitar a inserção da metodologia BIM, quando se trata do ensino de modelagem de projetos de instalações elétricas.

Palavras-chave: Building Information Modeling. Ensino. Modelagem 3D. 


\section{BIM TEACHING: EXPERIENCE IN ELECTRICAL INSTALLATIONS DESIGNS IN IFRN}

Abstract:

Federal Decree No. 10,306 of April 2, 2020 increased the urgency to implement Building Information Modeling (BIM) in the market of Architecture, Engineering, Construction and Operation (AECO) in the country. This reality directly implies the academy, which needs to attend the training of professionals in BIM. However, courses in the area already have a curricular matrix with a defined course load. In this context, an academy faces difficulties to adapt the need to insert BIM content, without compromising the other contents. Given this scenario, distance learning platforms can be presented as an ally. The article aims to verify the perception of students of the Federal Institute of Education, Science and Technology of Rio Grande do Norte (IFRN) "Buildings course" when experimenting the educational scenario for the insertion of BIM. The research method adopted was the case study, with a time frame in the year 2019, and the unit of analysis was the Clube do Instalador event, from the IFRN, which counts with the participation of students in the Buildings course. The results indicate that the use of technological teaching tools allow young students inserted in this scenario to present better performance in learning of academic activities. In this context, it is possible to conclude that the application of the proposed educational scenario, with the help of a virtual learning environment (AVA) has the potential to facilitate the insertion of the BIM methodology, when dealing with the teaching of modeling of electrical installations designs.

Keywords: Building Information Modeling. Teaching. 3D modeling.

\section{INTRODUÇÃO}

O Building Information Modeling (BIM) é um conjunto inter-relacionado de políticas, processos e tecnologias, capazes de produzir, utilizar ou atualizar um modelo virtual de informações da edificação (SUCCAR, 2009). Essa ampla abrangência do BIM é o que garante as inúmeras vantagens já detectadas quando da sua implementação, entre as quais pode-se destacar a modelagem paramétrica, que permite alterações nos componentes modelados, com a geração de atualizações instantâneas em todo o projeto, resultando em redução de conflitos, facilidade de revisões e consequentemente aumento de produtividade (FLORIO, 2007).

Diante desse quadro, o governo federal, associações e entidades vêm buscando incentivar a implementação do BIM no país. Entre as iniciativas, pode-se elencar o Guia de Boas Práticas BIM (AsBEA, 2013), a Coletânea de Guias para implementação do BIM em construtoras e incorporadoras (CBIC, 2016) e a Coletânea Guias BIM ABDI-MDIC (ABDI, 2017). De forma mais formal, tem-se o decreto federal de 5 de junho de 2017, que instituiu o Comitê Estratégico de Implementação do Building Information Modelling - CE-BIM, de caráter temporário e objetivando propor a Estratégia Nacional de Disseminação do BIM (BRASIL, 2017) e, mais recentemente, o decreto federal $n^{\circ} 10.306$ de 02 de abril de 2020, que estabeleceu a utilização do BIM na execução direta e indireta de obras e serviços de engenharia realizadas pelos órgãos e pelas entidades da administração pública federal (BRASIL, 2020).

No entanto, mesmo diante dessa urgência de profissionais habilitados e com experiência em BIM, a academia tem enfrentado dificuldades para implementar o BIM em seus cursos da área de Arquitetura, Engenharia, Construção e Operação (AECO). Santos (2017), identificando como o BIM vem sendo incorporado em cursos de Arquitetura e Urbanismo, concluiu que a 
incorporação do BIM ainda é incipiente. Quando de trata de cursos técnicos de nível médio a situação é mais crítica ainda, sendo poucas as pesquisas. Há que se destacar trabalhos que buscam o diagnóstico da permeabilidade do BIM na matriz curricular do curso de Edificações (CUPERSCHMID; CRUZ; RUSCHEL, 2017; GIESTA; MENEZES; COSTA NETO, 2017) e o trabalho que propõe o ensino do BIM para alunos de Edificações pelo viés da Iniciação Científica (GIESTA; COSTA; COSTA NETO, 2019).

Experiências vêm mostrando que tecnologias digitais, ensino híbrido e sala de aula invertida podem ser opções para facilitar a inserção do BIM na academia. Leal (2018) apresenta possibilidades de uso de Tecnologias de Informação e Comunicação (TICs) para o ensino em Arquitetura e Urbanismo, objetivando a motivação dos alunos de forma a permitir um maior aprendizado. Moran (2013, apud SILVA; KALHIL, 2018, p. 85) aponta que "as tecnologias digitais facilitam a pesquisa, a comunicação e a divulgação em rede". Cuperschmid; Castriotto (2018) discorrem sobre a possibilidade de disponibilização de vídeos tutoriais para aprendizagem de ferramentas BIM, em estratégia de sala de aula invertida (ensino híbrido).

Nesse sentido, identifica-se a carência de estudos que abordem o uso de TICs para o ensino de BIM para alunos de Edificações, representando uma lacuna a ser preenchida. Assim, o presente artigo tem como objetivo contribuir nessa seara, verificando a percepção de alunos do curso de Edificações do IFRN em experimentação de cenário educacional com a utilização de TICs para inserção do BIM.

\section{MÉTODO}

O método utilizado na pesquisa foi o estudo de caso, que para Ventura (2007) é entendido como uma metodologia ou como a escolha de um objeto de estudo definido pelo interesse em casos individuais. Visa à investigação de um caso específico, bem delimitado, contextualizado em tempo e lugar para que se possa realizar uma busca circunstanciada de informações. Segundo Gil (2002, p. 54), "para a realização de estudos de caso não são definidos procedimentos metodológicos rígidos". Nesta pesquisa, foram definidas cinco etapas que mostram o seu delineamento: a) delimitação da unidade; b) preparação; c) coleta de dados; d) análise dos dados; e) elaboração do relatório (Figura 1).

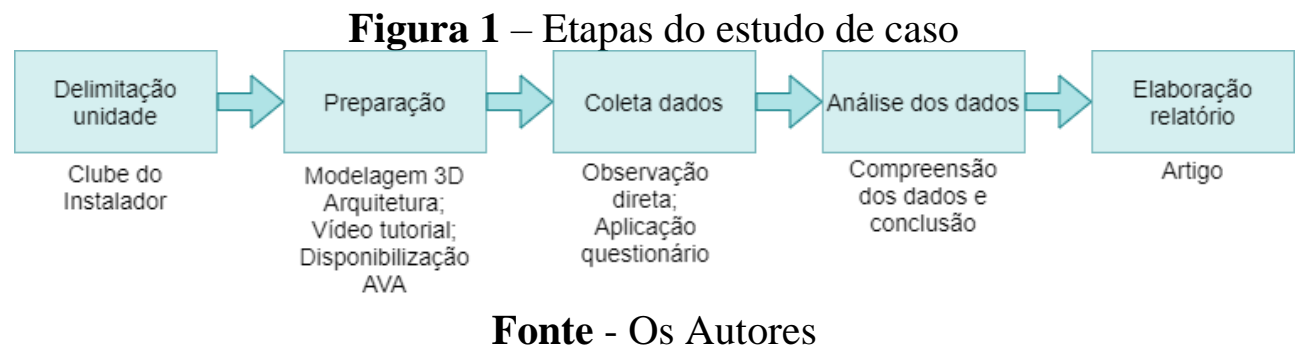

Na etapa de delimitação da unidade, foi definida a unidade de análise, que correspondeu à quarta fase do Clube do Instalador, no ano de 2019. O Clube do Instalador é uma nova estratégia educacional, que tem como meta melhorar o processo de aprendizagem, abrangendo as disciplinas de 'Instalações Elétricas', 'Instalações Hidrossanitárias' e 'Instalações de Segurança', do currículo do curso de Edificações do IFRN, nas modalidades Integrado e Subsequente. Com ênfase no desenvolvimento de habilidades teóricas e práticas, o Clube do Instalador tem como mecanismo de avaliação uma competição, cujo método se refere à análise do índice de desempenho nas provas, em cada uma das 6 fases do evento.

A etapa de preparação se constituiu na modelagem 3D da disciplina arquitetura, de uma residência de $80 \mathrm{~m}^{2}$ (Figura 2). Para tanto, foi escolhido o software Autodesk Revit (2019), em 
razão de ser o existente no laboratório do Grupo de Estudos e Pesquisa em Integração de Projetos (GIP), bem como já ser de conhecimento dos pesquisadores. Ainda nessa etapa, foi realizada a preparação de um vídeo tutorial com o passo a passo para a modelagem de um projeto de instalações elétricas no software BIM. Na sequência, esse vídeo foi disponibilizado aos alunos, através da plataforma Modular Object-Oriented Dynamic Learning Environment (MOODLE), com antecedência, de modo a que os mesmos pudessem aprender a utilizar a ferramenta. A plataforma MOODLE é um Ambiente Virtual de Aprendizagem (AVA) que dispõe de ferramentas que possibilitam gerenciar uma disciplina ou um curso, potencializando o processo ensino/aprendizagem mediado pelas TICs (IFF, 2020).

Figura 2 - Projeto arquitetônico para o desenvolvimento do projeto de instalações elétricas

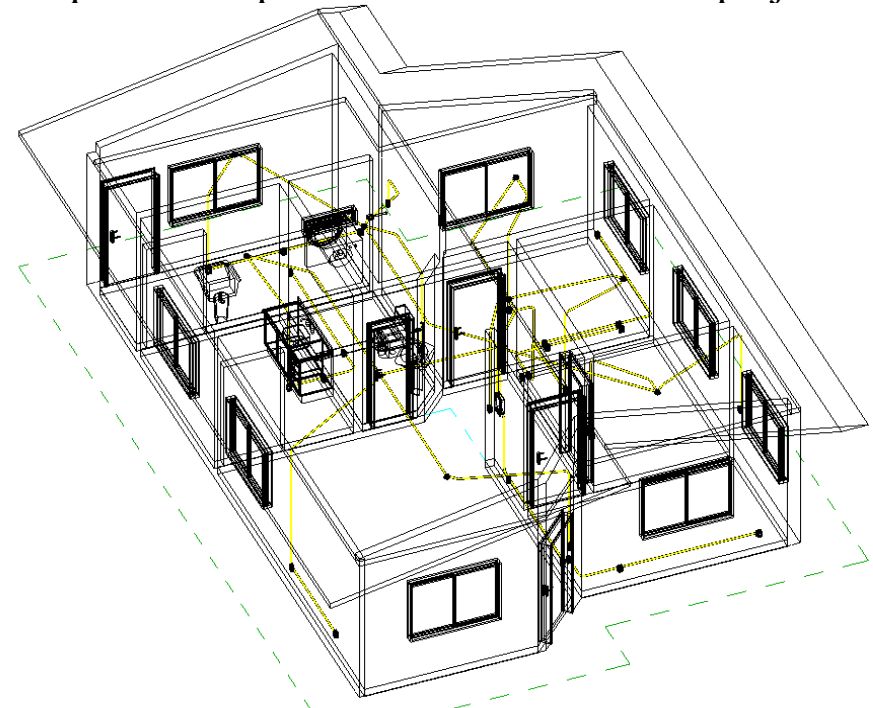

Fonte - Os Autores

A etapa de coleta de dados foi realizada através de duas técnicas: a) aplicação de um questionário com perguntas fechadas, que segundo Dresch; Lacerda e Antunes Júnior (2015) é a categoria que apresenta alternativas para o respondente, restringindo as respostas, ao mesmo tempo em que facilita a análise dos dados, por sua objetividade; e b) observação direta, técnica essa que permite ao investigador identificar certas características do fenômeno ou sistema em estudo que muitas vezes passam despercebidas pelos indivíduos que fazem parte desse sistema (DRESCH; LACERDA; ANTUNES JÚNIOR, 2015).

A observação direta se deu quando do desenvolvimento e modelagem do projeto elétrico por parte dos participantes e através da análise do projeto modelado. Nesse momento, um grupo de 10 alunos, os quais foram divididos em duplas, tiveram um tempo máximo de 3 horas para o desenvolvimento do projeto elétrico, sua modelagem, bem como para a extração dos quantitativos. Com relação ao projeto elétrico, é importante destacar que o mesmo se caracterizou como de dificuldade elevada, visto que consistiu em: Dimensionamento da potência de iluminação; Dimensionamento da quantidade e potência das Tomadas de uso Geral (TUGs) e das Tomadas de Uso Específico (TUEs); Definição dos comandos dos interruptores; Criação dos circuitos para TUGs, TUEs e iluminação; Lançamento dos eletrodutos de modo a atender todos os pontos; Identificações das fiações; Identificações de potência, número do circuito, pontos de luz e comandos de interruptores.

Após a conclusão dessas atividades, os alunos responderam ao questionário, composto por 8 perguntas, dividido em 3 eixos: a) análise do processo de aprendizagem utilizado; b) análise do software BIM; c) análise do processo ensino de software BIM aplicado. O eixo de análise do processo de aprendizagem utilizado foi composto por 4 perguntas, objetivando identificar 
como os alunos se relacionaram com a plataforma MOODLE e com o vídeo tutorial. O eixo de análise do software BIM buscou compreender a visão dos alunos com relação ao software utilizado e teve 2 perguntas. No eixo de análise do processo ensino de software BIM, composto por 2 perguntas, os alunos foram questionados com relação à viabilidade da utilização da plataforma para o ensino do software BIM.

$\mathrm{Na}$ quarta etapa do estudo de caso, as análises foram realizadas, de forma a permitir a etapa final de conclusão, com a elaboração de um artigo.

\section{ANÁLISE DOS DADOS}

A análise dos dados (Figura 3) mostrou que $70 \%$ dos alunos identificou a aplicação da plataforma MOODLE como de fácil acesso, indicando sua grande possibilidade de utilização.

Figura 3 - Questionário e respostas

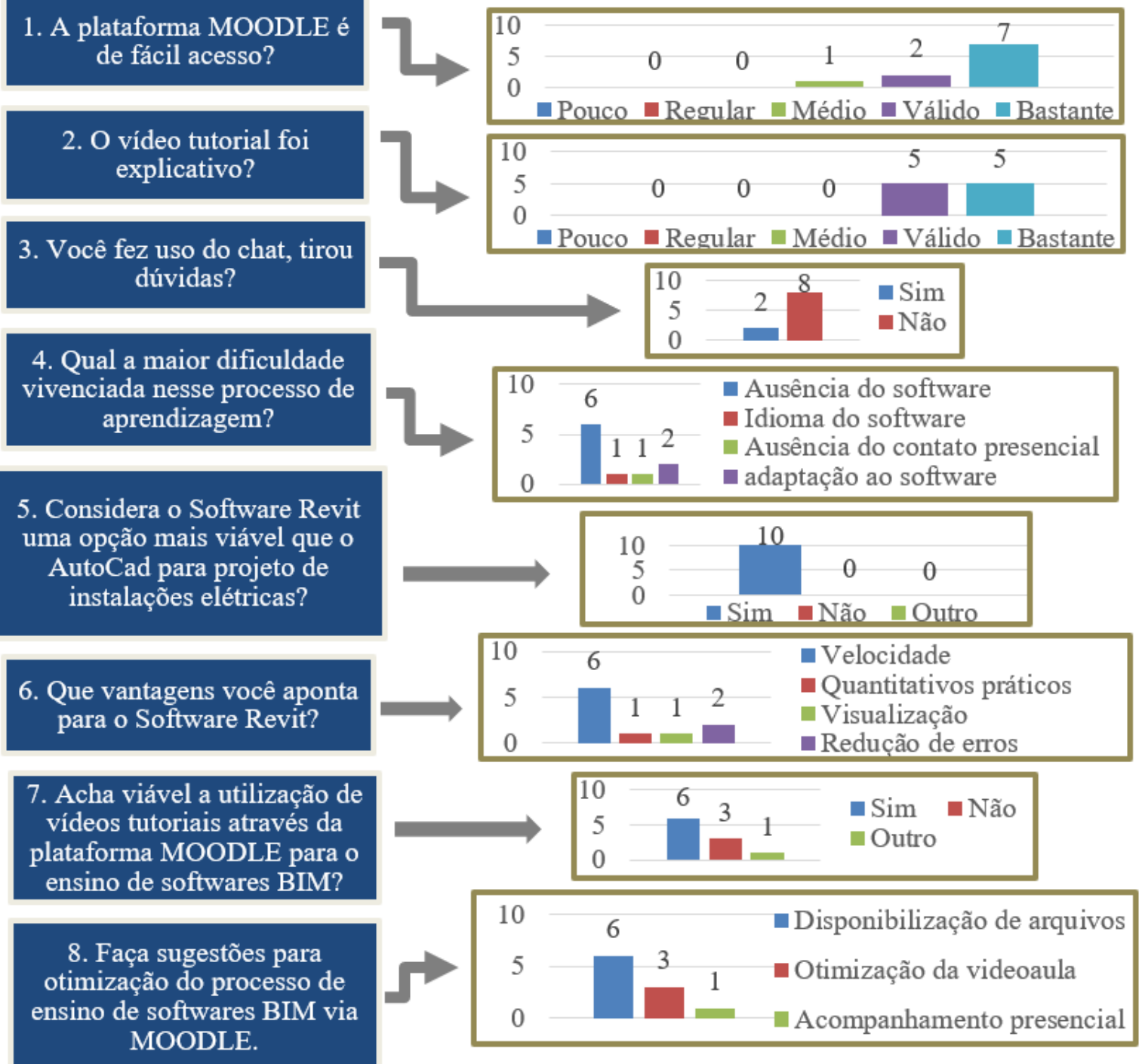

Fonte - Os Autores

Com relação à disponibilização de chat, apenas $20 \%$ dos alunos fizeram uso. Sobre as dificuldades vivenciadas no processo de aprendizagem, 60\% dos participantes destacou a ausência de software para treinar o passo a passo apresentado no vídeo tutorial como a mais 
relevante. Ainda nesse eixo, 50\% dos alunos apontou o vídeo tutorial como bastante explicativo e os outros $50 \%$ como válido, indicando que todos os alunos consideram viável o ensino do software BIM através de vídeos tutoriais.

Com relação aos dados do segundo eixo, tem-se que todos os alunos consideraram o software BIM analisado mais viável que o AutoCAD para elaboração de projeto de instalações elétricas, bem como, quando questionados sobre as vantagens do software em questão, $60 \%$ dos participantes apontou a velocidade de desenvolvimento do projeto como a maior vantagem.

No terceiro eixo, $60 \%$ dos entrevistados destacou a viabilidade da utilização de vídeos tutoriais através da plataforma MOODLE para o ensino do software BIM. Ainda nesse eixo, $60 \%$ apresentou a disponibilização prévia dos arquivos para treino como sugestão para otimização do processo de ensino de software BIM no formato utilizado.

A análise dos projetos modelados pelos alunos (Gráfico 1) apontou que todas as duplas obtiveram um desempenho superior a $70 \%$, e que $80 \%$ das duplas alcançou mais de $80 \%$, com uma dupla tendo $100 \%$. Tais resultados corroboraram com os alcançados nos questionários, que indicaram que a maioria dos alunos considerou a plataforma utilizada de fácil acesso, que o vídeo tutorial estava bem explicativo, motivo pelo qual não necessitaram tirar dúvidas pelo chat, bem como que consideraram a estratégia uma boa solução para o processo de implementação do BIM na academia.

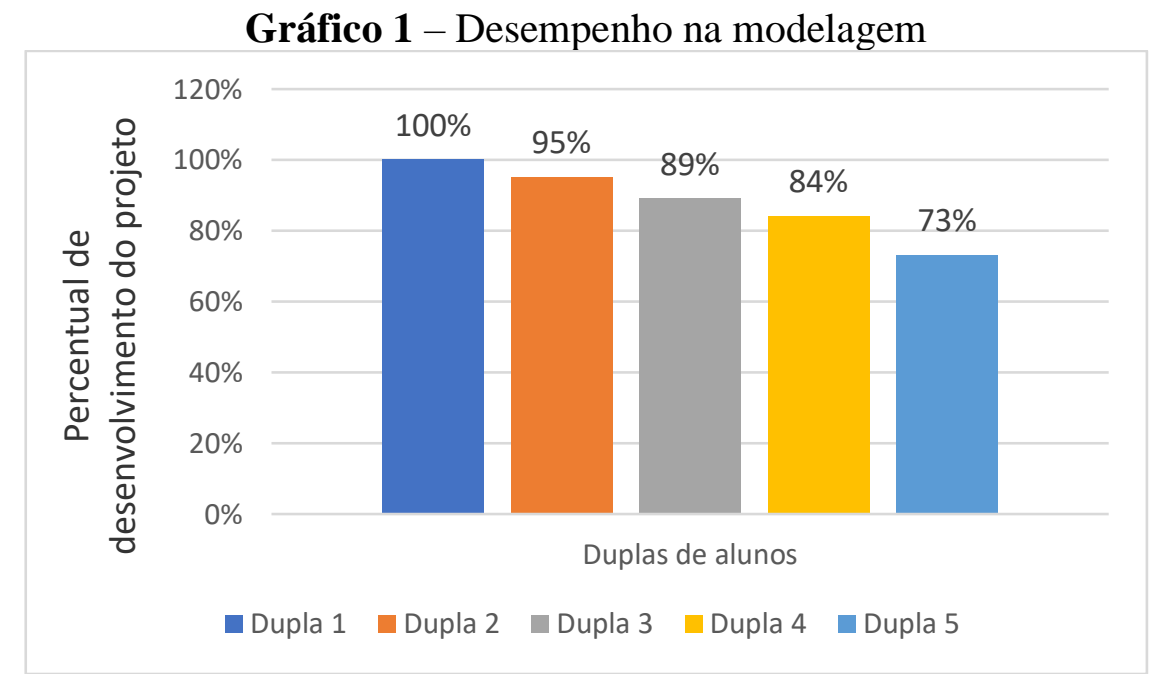

Fonte - Os Autores

\section{CONCLUSÃO}

Objetivando verificar a percepção de alunos do curso de Edificações do IFRN em experimentação de cenário educacional para inserção do BIM, utilizando vídeo tutorial disponibilizado no Ambiente Virtual de Aprendizagem (AVA) Modular Object-Oriented Dynamic Learning Environment (MOODLE), foi realizado um estudo de caso no Clube do Instalador do IFRN. Como resultado, observou-se um grande potencial da utilização de vídeos tutoriais em plataformas como a MOODLE para o ensino de softwares BIM para projetos de instalações elétricas. Esses resultados foram alcançados tanto através da análise dos questionários aplicados com os alunos, como através da avaliação da qualidade da modelagem desenvolvida por eles. 
Diante desse cenário, vislumbra-se que a utilização de um AVA e o ensino de softwares através de vídeos tutoriais podem representar uma solução para o processo de implementação do BIM nos cursos da área de AECO, sem comprometer a matriz curricular existente.

Como limitações, pode-se apontar a experimentação ter sido específica para a modelagem de projeto de instalações elétricas e o alto nível dos alunos utilizados no estudo de caso, visto que os mesmos fazem parte de um grupo seleto de 10 alunos, que passaram para a $4^{\text {a }}$ fase do Clube do Instalador, a qual contou com 343 participantes, envolvendo 4 campi do Rio Grande do Norte. Nesse contexto, tem-se como proposta para pesquisas futuras que a experimentação seja feita em uma turma de alunos de Edificações, em sala de aula tradicional.

\section{AGRADECIMENTOS}

Os autores agradecem à DIPEQ - Diretoria de Pesquisa e Inovação, ao IFRN Campus Natal Central e ao evento Clube do Instalador do Instituto Federal de Educação, Ciência e Tecnologia do Rio Grande do Norte (IFRN) pelo apoio e recepção da pesquisa desenvolvida.

\section{REFERÊNCIAS}

BRASIL. Decreto de 05 de junho de 2017. Dispõe sobre a instituição do Comitê Estratégico de Implementação do Building Information Modelling. Diário Oficial da República Federativa do Brasil, Brasília, n. 107, Seção 1, p. 19, jun. 2017.

BRASIL. Decreto $n^{\circ}$ 10.306, de 02 de abril de 2020. Dispõe sobre a utilização do Building Information Modelling - BIM ou Modelagem da Informação da Construção na execução direta ou indireta de obras e serviços de engenharia, realizada pelos órgãos e pelas entidades da administração pública federal, no âmbito da Estratégia Nacional de Disseminação do Building Information Modelling - Estratégia BIM BR. Diário Oficial da República Federativa do Brasil, Brasília, n. 65, Seção 1, p. 5-7, abr. 2020.

CUPERSCHMID, Ana Regina Mizrahy; CASTRIOTTO, Caio Magalhães; Teaching BIM modeling in the architecture course: using a Blended Learning Strategy, p. 942-947. São Paulo: Blucher, 2018. ISSN 2318-6968, DOI 10.5151/sigradi2018-1245

CUPERSCHMID, A.; CRUZ, M. O.; RUSCHEL, R. C. A incorporação de BIM no ensino do curso Técnico em Edificações. Gestão \& Tecnologia de Projetos, São Carlos, v. 12, n. 2, p. 117-134. 2017. http://dx.doi.org/10.11606/gtp. v12i2.131498

DRESCH, A.; LACERDA, D. P.; ANTUNES Jr., J. A. V. Design Science Research: Método de Pesquisa para Avanço da Ciência e Tecnologia. Porto Alegre: Bookman, 2015. 204 p.

FLORIO, W. Contribuições do Building Information Modeling no processo de projeto em arquitetura. Anais... In: $3^{\circ}$ ENCONTRO DE TECNOLOGIA E COMUNICAÇÃO DA CONSTRUÇÃO CIVIL. Porto Alegre, RS: 2007.

GIESTA, J. P; COSTA, T. G.; COSTA NETO, A. Inserção do ensino do Building Information Modeling (BIM) na Academia: novas perspectivas por meio da pesquisa. In: SIMPÓSIO BRASILEIRO DE TECNOLOGIA DA INFORMAÇÃO E COMUNICAÇÃO NA CONSTRUÇÃO, 2., 2019, Campinas, SP. Anais[...]. Porto Alegre: ANTAC, 2019. Disponível em: https://antaceventos.net.br/index.php/sbtic/sbtic2019/paper/view/176 
GIESTA, J. P.; MENEZES, G. L. B. B.; COSTA NETO, A. Integração do BIM no currículo do curso de Edificações. In: SIMPÓSIO BRASILEIRO DE TECNOLOGIA DA INFORMAÇÃO E COMUNICAÇÃO NA CONSTRUÇÃO, 1., SIMPÓSIO BRASILEIRO DE GESTÃO E ECONOMIA DA CONSTRUÇÃO, 10., 2017, Fortaleza. Anais[...].Porto Alegre: ANTAC, 2017.

GIL, A. C. Como elaborar projetos e pesquisa. 4. ed. São Paulo: Atlas; 2002. 175 p.

Instituto Federal Fluminense (IFF). Curso de Formação Continuada em Metodologias Ativas de Ensino. Rio de Janeiro: IFF, 2020.

LEAL, B. M. F. Propostas para o ensino dos conteúdos de Arquitetura e Urbanismo através de ferramentas digitais. 2018. 191 p. (Mestrado em Ciências em Arquitetura) Faculdade de Arquitetura e Urbanismo, Universidade Federal do Rio de Janeiro, Rio de Janeiro, 2018.

MORAN, J. M. Desafios que as tecnologias digitais nos trazem. In: MORAN, J. M. Novas tecnologias e mediação pedagógica. Campinas, São Paulo: Papirus, 2013.

SANTOS, L. A. dos. Building Information Modeling no Ensino de Arquitetura e Urbanismo: Percepção e disseminação do BIM nas Instituições de Ensino Superior do Estado de São Paulo. 2017. 138 f. Dissertação (Mestrado em Arquitetura e Urbanismo) - Universidade São Judas Tadeu, São Paulo, 2017.

SILVA, Wender Antônio da; KALHIL, Josefina Barrera. Tecnologias digitais no ensino de ciências: reflexões e possibilidades na construção do conhecimento científico. Revista Brasileira de Educação em Ciências e Educação Matemática, 2018, 2.1: 77-91.

SUCCAR, B. Building Information Modelling Framework: a research and delivery foundation for industry stakeholders. Automation in Construction, v. 18, n. 3, p. 357-375, 2009.

VENTURA, Magda Maria. O estudo de caso como modalidade de pesquisa. Revista SoCERJ, 2007, 20.5: 383-386. 